\title{
Electroporation of RNA stimulates immunity to an encoded reporter gene in mice
}

\author{
JOHN M. PIGGOTT ${ }^{1,2}$, BRIAN J. SHEAHAN ${ }^{3}$, DECLAN M. SODEN ${ }^{2}$, \\ GERALD C. O'SULLIVAN ${ }^{2}$ and GREGORY J. ATKINS ${ }^{1}$
}

\author{
${ }^{1}$ Virus Group, Department of Microbiology, School of Genetics and Microbiology, The Moyne Institute of \\ Preventive Medicine, Trinity College, Dublin 2; ${ }^{2}$ Cork Cancer Research Centre, Biosciences Institute, \\ National University of Ireland, Cork; ${ }^{3}$ Veterinary Sciences Centre, UCD School of Agriculture, \\ Food Science and Veterinary Medicine, University College Dublin, Belfield, Dublin 4, Ireland
}

Received May 11, 2009; Accepted June 17, 2009

DOI: $10.3892 / \mathrm{mmr} 00000168$

\begin{abstract}
Electroporation is the application of high-voltage short-duration pulses to transiently permeabilize cells, permitting the cellular uptake of macromolecules, including nucleic acid. Although much attention has been focused on DNA vaccines, antigen-encoding RNA molecules may also stimulate immunity. Several methods are being examined in an effort to enhance the efficacy of nucleic acid delivery. One such method is the application of electroporation. The present study was designed to develop electroporation for use as a method of RNA delivery in conjunction with the Semliki Forest virus (SFV) RNA vector system for stimulation of immunity. Expression of SFV-based $\beta$-galactosidase and luciferase vectors was observed in the muscle after electroporation. Although some tissue damage was induced following intramuscular injection and electroporation with SFV vector RNA encoding LacZ at optimum pulse conditions, immunity to LacZ was efficiently induced. Following two immunizations, there was a higher IgG2a antibody response with the viral vector delivery and a higher IgG1 response in electroporated rSFV-LacZ RNA immunized mice.
\end{abstract}

\section{Introduction}

Antigen-encoding DNA molecules incorporating a eukaryotic promoter (DNA vaccines) have been shown to induce both cell-mediated and humoral immunity (1), but a DNA vaccine must be delivered to the nucleus before it can be transcribed and translated to express antigen. This requires efficient membrane and nuclear permeabilization to allow the DNA

Correspondence to: ProfessorG.J. Atkins, Virus Group, Department of Microbiology, School of Genetics and Microbiology, The Moyne Institute of Preventive Medicine, Trinity College, Dublin 2, Ireland E-mail: gatkins@tcd.ie

Key words: nucleic acid immunization, electroporation, Semliki Forest virus, vector, RNA vaccines to enter the cells (2). This is not the case with RNA molecules, where it may only be necessary to deliver the gene through the cell membrane. In the case of Semliki Forest virus (SFV) vectors, the RNA replicates and is expressed only in the cytoplasm (3). Although recombinant virus-like particles (VLPs) are the most commonly used SFV vector system, it has been shown that naked RNA molecules based on the SFV vector system as well as encoding heterologous antigens can stimulate immunity, although less efficiently than VLPs (4).

Electroporation increases the effectiveness of nonviral gene therapy and could enhance the delivery of nucleic acids to the cytoplasm. It substantially increases DNA delivery and DNA vaccine potency, and appears to be well tolerated (5). This has been shown for reporter genes and genes for therapeutic applications, such as erythropoietin (6) and herpes thymidine kinase (7).

While high levels of gene expression are required following transfection by electroporation, it is also desirable to minimize tissue damage. Tissue damage is an important adverse effect of electroporation and can affect gene transfer by reducing the number of viable cells that express the gene. Electroporation parameters have been shown to influence tissue damage and also gene expression (and hence antibody titers), so highvoltage short-pulse duration conditions are typically used (7). However, these parameters are based on experiments with DNA, while RNA has not yet been tested.

Among the tissues targeted for electroporation with DNA are tumors $(8)$, skin $(9,10)$ and muscle $(5,10-12)$. In this study, we showed that antigen expression following electroporative delivery of an RNA vector into muscle significantly increases the efficiency of expression and subsequent immune stimulation in mice.

\section{Materials and methods}

Mice. Specific pathogen-free 4- to 6-week-old female BALB/c mice used in the study were obtained from Harlan, UK and maintained in accordance with the principles outlined in the S1 17/94 European Community Regulations 1994 for the Care and Use of Laboratory Animals. All experiments were 
performed after approval of protocol and under license. Blood was harvested from mice from the facial vein using a 28 -gauge needle on days $0,7,13,20$ and 26 after inoculation. The blood was incubated at $4^{\circ} \mathrm{C}$ for $12 \mathrm{~h}$ to allow coagulation and then centrifuged at 10,000 rpm for $10 \mathrm{~min}$. The serum was decanted and aliquoted before storage at $-70^{\circ} \mathrm{C}$.

Plasmids. The SFV vector-encoding plasmids pSFV-LacZ, pSFV-EGFP and helper vectors pSFV-SP6-helper-S2 (encoding SFV envelope proteins) and pSFV-SP6-CS219A (encoding SFV capsid protein) were a gift from Professor P. Liljeström, Karolinska Institute, Stockholm, Sweden and were used in the preparation of SFV recombinant VLPs (13). The SFV luciferase vector-encoding plasmid was a gift from Dr C. Smerdou, University of Navarra, Pamplona, Spain. The RNA transcribed from such plasmids was labeled rSFV-X, where $\mathrm{X}$ is the transgene. RNA was transcribed from the plasmids using the SP6 promoter as previously described (13), and each was adjusted to a concentration of $1 \mu \mathrm{g} / \mu \mathrm{l}$ with nuclease-free water. Mice were anesthetized using a xylazine/ketamine $(0.1 \mathrm{ml} / 10 \mathrm{~g})$ mixture. Groups of mice to be immunized received a $50-\mu \mathrm{l}$ intramuscular (i.m.) injection of the RNA at a concentration of $1 \mu \mathrm{g} / \mu \mathrm{l}$ in the left tibialis anterior muscle.

Electroporation. The electroporation needles (28-gauge) were inserted to either side of the injection site $(0.5 \mathrm{~cm}$ apart), and after 1-2 min the muscle was electroporated with six squarewave pulses at $400 \mathrm{~V} / \mathrm{cm}$ of $10-\mathrm{msec}$ duration (with a 1-sec pulse interval) using the Cliniporator electroporation device (Igea, Italy). Mice were boosted 13 days later under the same conditions, but in the right tibialis anterior muscle. Groups of control mice were simultaneously injected with RNA alone (no electroporation) or electroporated without nucleic acid.

Immunization with VLPs. rSFV-LacZ VLPs were prepared as described by Smerdou and Liljeström (13) and adjusted to a concentration of $10^{6}$ infectious units (IU)/ml in TNE buffer. The groups of mice to be immunized received a $50-\mu 1$ i.m. injection of the rSFV-LacZ VLPs in TNE at a concentration of $10^{6} \mathrm{IU} / \mathrm{ml}$ in the left tibialis anterior muscle, and were boosted 14 days later with the same concentration in the right tibialis anterior muscle. Groups of control mice were simultaneously immunized with TNE alone or rSFV-Luc/EGFP VLPs in the same manner. Six mice were used per treatment group.

Measurement of antibody response. $\beta$-galactosidase (Roche) was used as a reporter gene for analysis of antibody responses following electroporation. Sera from blood samples collected on days $0,7,13,20$ and 26 from inoculated and boosted $\mathrm{BALB} / \mathrm{c}$ mice were analyzed using ELISA. A flat-bottomed 96-well ELISA plate was coated with $2.5 \mu \mathrm{g} / 100 \mu \mathrm{l}$ of $\beta$-galactosidase $(100 \mu \mathrm{l} /$ well $)$, and the serum was added in serial dilutions. The plate was stored at room temperature for $1 \mathrm{~h}$, washed, coated with secondary antibody (50 $\mu \mathrm{l}$ of $1 / 500$ dilution anti-mouse HRP, BD Biosciences) and incubated in the dark at room temperature for $1 \mathrm{~h}$. Following a washing step, 3,3',5'5-tetramethylbenzidine (Sigma, $100 \mu \mathrm{l} /$ well) was added to the plate. After $4 \mathrm{~min}$, the stopping buffer $(50 \mu \mathrm{l} /$ well) was added. The absorbance was read at $450 \mathrm{~nm}$, and the mean antibody titers calculated.
Histopathology. For the preparation of muscle cryosections, excised muscle was embedded in OCT compound (TissueTec, USA) on cork disks (Lamb, UK), snap-frozen in liquid nitrogencooled isopentane and stored at $-70^{\circ} \mathrm{C}$. A Leica CM 1900 cryostat was used to prepare $4-$ to $6-\mu \mathrm{m}$ sections at $-15^{\circ} \mathrm{C}$, which were placed on poly-L-lysine-coated glass slides, fixed in acetone at $-20^{\circ} \mathrm{C}$ for $5 \mathrm{~min}$, air-dried overnight and stored in airtight containers at $-70^{\circ} \mathrm{C}$. Muscle sections were stained with hematoxylin and eosin.

Transfection efficiency. Mice were anesthetized using a xylazine/ketamine mixture $(0.1 \mathrm{ml} / 10 \mathrm{~g}) 48 \mathrm{~h}$ after luciferase transfection. For electroporation, $50 \mu \mathrm{l}$ of RNA $(1 \mu \mathrm{g} / \mu \mathrm{l})$ was used, and for luciferase VLPs, $10^{6} \mathrm{IU} / \mathrm{ml}$. Luciferase reagent $(100 \mu \mathrm{l})$ (Promega) was delivered i.p., and the mice were examined after 3 min using a Xenogen In Vivo Imaging System.

Statistical analysis. The t-test was used to analyze the normally distributed data of two groups when the standard deviations were similar. For more than two groups, the ANOVA/Tukey test was used. A p-value $<0.05$ was considered significant.

\section{Results and Discussion}

Optimization of electroporation. Preliminary experiments for intramuscular electroporation showed that $50 \mu \mathrm{g}$ of RNA was optimal for expression $48 \mathrm{~h}$ post transfection (Fig. 1A and B). At voltages $<200$ or $>600 \mathrm{~V} / \mathrm{cm}$, no reporter gene expression was observed, while optimal expression following electroporation was observed at $400 \mathrm{~V} / \mathrm{cm}$ (Fig. 1C). The optimal pulse length was $10 \mathrm{msec}$ (Fig. 1D). Three, six and eight pulses (with a 1-sec interval) were also examined, but no increased transfection was observed for eight compared to six pulses (data not shown).

Tissue damage. The level of tissue damage was examined by histological analysis of $4-\mu \mathrm{m}$ sections taken from the muscle under optimal electroporation conditions. The damage was not obvious immediately following electroporation, but was observed at $72 \mathrm{~h}$ post electroporation. There was no evidence of tissue damage 7 days following electroporation. While necrosis, swelling and needle marks were noted in almost all cases, hemorrhaging was observed in $30 \%$ of the cases. Tissue damage was also examined using a range of different voltages (Table I). The necrotic cells exhibited pyknosis of the nucleus and amorphous cytoplasmic eosinophilia. As shown in Fig. 1D and E, advanced necrosis was characterized by nuclear karyorrhexis, karyolysis and cytolysis. A decrease in tissue damage was observed when lower voltages were applied, but this resulted in a corresponding decrease in gene expression. At all voltage potentials $>100 \mathrm{~V} / \mathrm{cm}$, some degree of tissue damage was evident. As the voltage was increased, a proportional increase in leucocytic infiltration was observed. When voltages $>600 \mathrm{~V} / \mathrm{cm}$ were applied, massive necrosis and in some cases edema were evident up to 21 days post electroporation.

The degree of muscle damage due to electroporation was shown to be dependent on the pulse applied. Previous 
A.

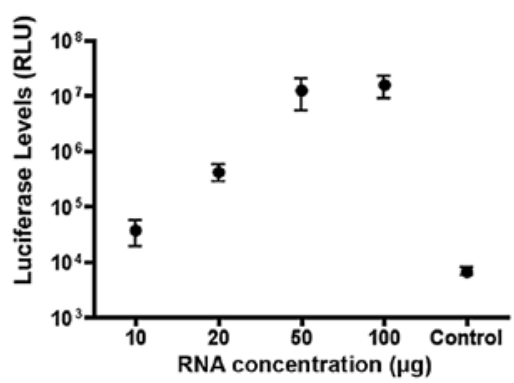

C.

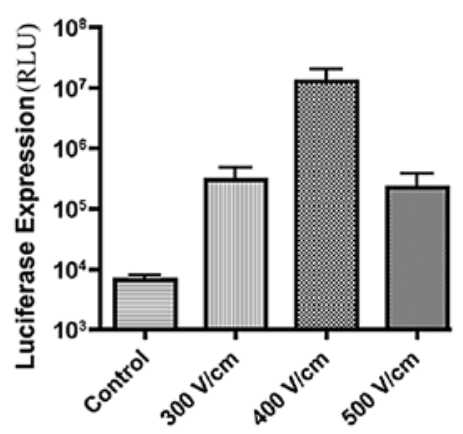

E.

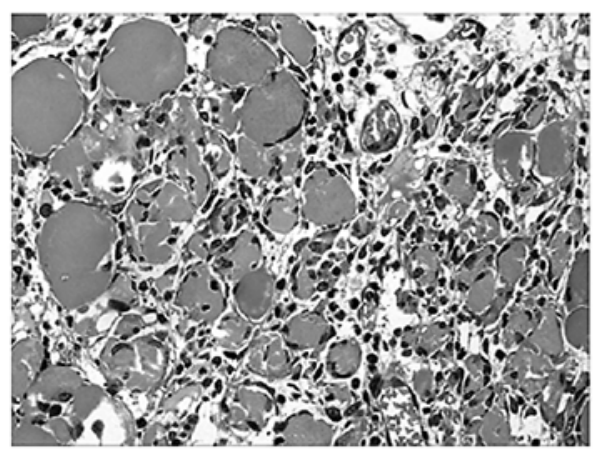

B.

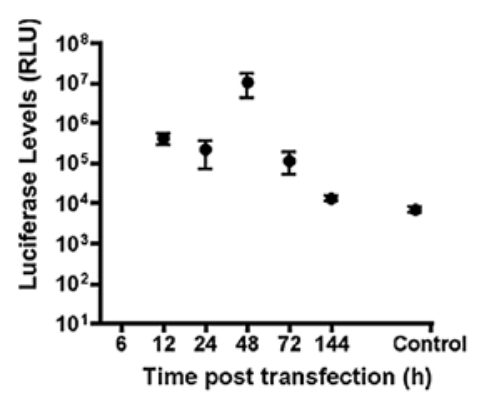

D.

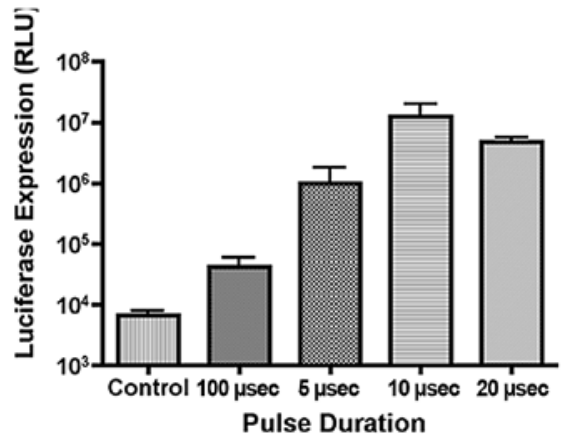

F.

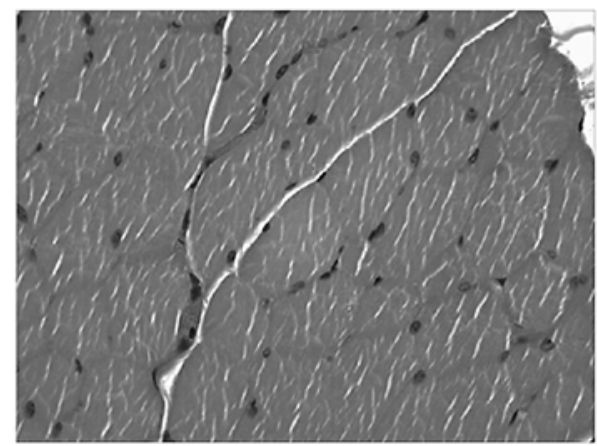

Figure 1. Parameters of electroporation. Luciferase levels were examined using the Xenogen live body imager at $48 \mathrm{~h}$ post electroporation. Luciferase expression (A) with varying concentrations of rSFV-Luc RNA $48 \mathrm{~h}$ after electroporation and (B) at intervals using $50 \mu \mathrm{g}$ rSFV-Luc following electroporation with six pulses of $400 \mathrm{~V} / \mathrm{cm}$ of 10 -msec duration. A control group with rSFV-Luc but without pulses was also included (no difference was observed, so only the value at $48 \mathrm{~h}$ is shown) $(\mathrm{n}=4)$. (C and D) Luciferase expression following intramuscular electroporation with rSFV-Luc (50 $\mu \mathrm{g})$ using varying voltages $(\mathrm{C})$ with six pulses of 10-msec duration and (D) with varying pulse lengths at $400 \mathrm{~V} / \mathrm{cm}$. In each set of experiments, a control group injected without pulse delivery was included (n=4). (E) Image of a $4-\mu \mathrm{m}$ section of muscle $48 \mathrm{~h}$ post electroporation with six pulses of $400 \mathrm{~V} / \mathrm{cm}$ of 10 -msec duration showing massive necrosis, edema, hemorrhage and low-grade leucocytic infiltration. There was no damage observed in the control non-electroporated section (F). (x200).

Table I. Damage induced in the muscle by electroporation. ${ }^{a}$

\begin{tabular}{lccccc}
\hline Voltage $(\mathrm{V} / \mathrm{cm})$ & Necrosis & Edema & Leucocytic infiltration & Calcification & Hemorrhage \\
\hline 0 & - & - & - & - & - \\
100 & + & - & + & - & - \\
250 & + & - & + & + & - \\
350 & ++ & + & ++ & + & + \\
400 & +++ & ++ & +++ & ++ & + \\
800 & +++ & & + & + \\
\hline
\end{tabular}

${ }^{a}$ Tissue damage was assessed at $48 \mathrm{~h}$ post electroporation $(\mathrm{n}=4)$. The severity of damage is denoted by,+++ and +++ corresponding to mild, moderate and severe damage detected in H\&E-stained sections (a minimum of twelve sections were analyzed per muscle for 6 mice). 
A.

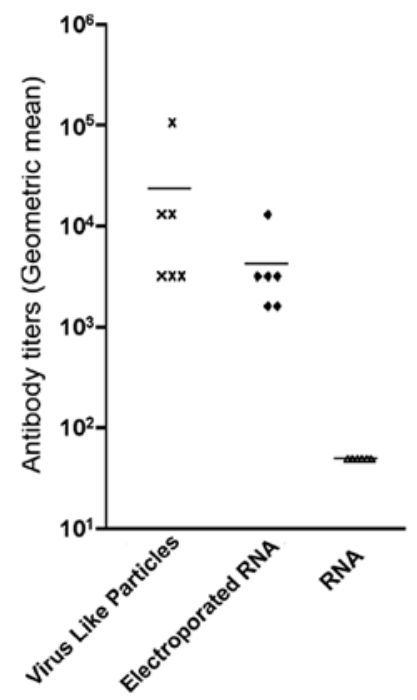

B.

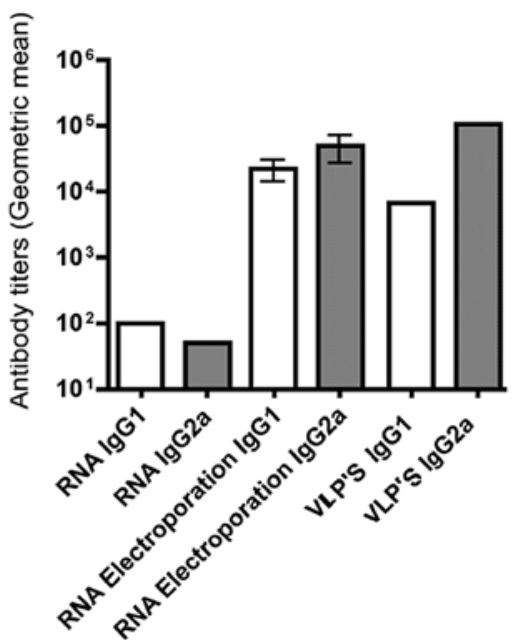

Figure 2. Serum antibody response following intramuscular delivery of a $\beta$-galactosidase reporter gene as rSFV-LacZ RNA or as rSFV-LacZ VLPs ( $\left.10^{6} \mathrm{IU}\right)$. Where electroporation was applied, there were six pulses of $400 \mathrm{~V} / \mathrm{cm}$ of 10-msec duration delivered 1-2 min following injection with the RNA (n=6). (A) Total IgG response. (B) Serum following two immunizations with the SFV-LacZ RNA or SFV VLPs encoding for LacZ was analyzed for the IgG isotype using ELISA.

data suggest that higher levels of tissue damage reduce transgene expression, but that this damage is reversible (7). Thus, a balance must be achieved between the induction of tissue damage and efficiency of transfection.

Antibody response. The effectiveness of the optimized intramuscular electroporation pulse conditions in combination with the SFV vector system was examined for the ability to induce an antibody response to $\beta$-galactosidase. Intramuscularly injected SFV RNA alone was compared to RNA followed by electroporation and SFV VLPs. Blood was taken 12 days following the first immunization, and antibody serum levels were analyzed using ELISA. The mice were subjected to a second immunization on day 13 and bled again on day 26 (Fig. 2A and B). There was a significant $(\mathrm{p} \leq 0.001)$ increase in the serum antibody levels when electroporation was applied following immunization with the $\beta$-galactosidase gene. There was also a significant increase in the serum antibody levels when mice were immunized with the SFV VLPs encoding LacZ. In all cases, an increase in antibody response was observed following a second immunization (Fig. 2A). There was a significantly higher IgG2a antibody response with the viral delivery and a significantly higher IgG1 response in the electroporated rSFV-LacZ RNA group (Fig. 2B).

This study demonstrated that electroporation promotes antibody responses induced by reporter RNA. Although the $400 \mathrm{~V} / \mathrm{cm}$ pulse has been shown to be optimal for this RNA electroporation system, the voltages used differ from other studies with DNA (7). However, we showed that RNA may be substituted for DNA for the induction of immune responses by electroporation, indicating that the use of RNA in combination with electroporation for applications such as vaccination should be further investigated. In terms of its solely cytoplasmic expression, the impossibility of insertional mutagenesis and the lack of dispersal (14), the use of RNA rather than DNA for this purpose may have advantages.

\section{References}

1. Donnelly J, Ulmer J, Shiver J and Liu M: DNA vaccines. Ann Rev Immunol 15: 617-648, 1997.

2. Nishikawa M and Huang L: Nonviral vectors in the new millennium: delivery barriers in gene transfer. Human Gene Ther 12: 861-870, 2001.

3. Strauss JH and Strauss EG: The alphaviruses: gene expression, replication, and evolution. Microbiol Rev 58: 491-562, 1994.

4. Fleeton MN, Chen M, Berglund P, Rhodes G, Parker SE, Murphy M, et al: Self-replicative RNA vaccines elicit protection against influenza A virus, respiratory syncytial virus, and a tickborne encephalitis virus. J Infect Dis 183: 1395-1398, 2001.

5. Widera G, Austin M, Rabussay D, Goldbeck C, Barnett SW, Chen M, et al: Increased DNA vaccine delivery and immunogenicity by electroporation in vivo. J Immunol 164: 635-640, 2000.

6. Rizzuto G, Cappelletti M, Maione D, Savino R, Lazzaro D and Costa P: Efficient and regulated erythropoietin production by naked DNA injection and muscle electroporation. Proc Natl Acad Sci USA 96: 6417-6422, 1999.

7. Wang XD, Tang JG, Xie XL, Yang JC, Li S, Ji JG and Gu J: A comprehensive study of optimal conditions for naked plasmid DNA transfer into skeletal muscle by electroporation. J Gene Med 7: 1235-1245, 2005.

8. Goto T, Nishi T, Tamura T, Dev SB, Takeshima H, Kochi M, et al: Highly efficient electro-gene therapy of solid tumour using an expression plasmid for the HSV-TK gene. Proc Natl Acad Sci USA 97: 354-359, 2000.

9. Titomirov AV, Sukharev S and Kistanova E: In vivo electroporation and stable transformation of skin cells of newborn mice by plasmid DNA. Biochim Biophys Acta 1088: 131-134, 1991.

10. Heller R, Coppola D, Pottinger C, Gilbert R and Jaroszeski MJ: Effect of electrochemotherapy on muscle and skin. Technol Cancer Res Treat 1: 385-391, 2002.

11. Selby M, Goldbeck C, Pertile T, Walsh R and Ulmer J: Enhancement of DNA vaccine potency by electroporation in vivo. J Biotech 83: 147-152, 2000.

12. Zucchelli S, Capone S, Fattori E, Folgori A, Di Marco A, Casimiro D, et al: Enhancing B- and T-cell immune response to a hepatitis C virus E2 DNA vaccine by intramuscular electrical gene transfer. J Virol 74: 11598-11607, 2000.

13. Smerdou C and Liljeström P: Two-helper RNA system for production of recombinant Semliki Forest virus particles. J Virol 73: 1092-1098, 1999.

14. Morris-Downes MM, Phenix KV, Smyth J, Sheahan BJ, Lileqvist S, Mooney DA, et al: Semliki Forest virus-based vaccines: persistence, distribution and pathological analysis in two animal systems. Vaccine 19: 1978-1988, 2001. 